Reprod. Nutr. Dévelop., 1983, 23 (2 B), 329-339.

\title{
Hyperglycémie maternelle et développement foetal chez le rat : conséquences d'une perfusion continue de glucose à la rate en fin de gestation
}

\author{
A. KTORZA, N. NURJHAN, J. R. GIRARD $\left({ }^{*}\right)$, L. PICON \\ avec la collaboration technique de Marie-France KINEBANYAN
}

Laboratoire de Physiologie du Développement, Laboratoire associé au C.N.R.S. $n^{\circ} 307$, Université Paris VII, 2, place Jussieu, 75251 Paris Cedex 05.

(*) Laboratoire de Physiologie du Développement, Collège de France, 11, place Marcelin Berthelot, 75231 Paris Cedex 05.

Summary. Maternal hyperglycemia and fetal development in rat : effects of continuous infusion of glucose to the rat in late pregnancy.

Continuous glucose infusion was used to induce mild hyperglycemia in unrestrained pregnant rats during the last three days of pregnancy. Control rats were infused with distilled water. Compared with the controls, fetuses from glucose-infused rats showed higher plasma glucose levels, increased plasma insulin and lower plasma glucagon concentrations. Pregnancy prolonged until day 23.5 resulted in a sharp decrease in plasma insulin concentrations and a dramatic increase in plasma glucagon concentrations. In 23.5-day old fetuses from both groups, plasma insulin concentration rose when phentolamine was injected but not when propanolol was injected. Plasma glucagon concentration in 23.5-day old fetuses from glucose-infused rats dropped with propanolol injection. In fetuses from control rats, liver phosphoenolpyruvate activity increased markedly and liver glycogen stores decreased sharply. In fetuses from glucose-infused rats, "liver phosphoenolpyruvate carboxykinase activity rose and glycogen content decreased, but to a lesser degree. Moreover, in postmature fetuses from glucose-infused rats, elevated plasma glucose and insulin concentrations were related to increased body weight and total carcass fat. Concurrently, the rate of lipogenesis in the carcass of these fetuses (estimated from the incorporation of ${ }^{3} \mathrm{H}$ from ${ }^{3} \mathrm{H}_{2} \mathrm{O}$ into fatty acids) was significantly increased.

\section{Introduction.}

Les effets de l'hyperglycémie maternelle sur la croissance du fotus et sur les sécrétions endocrines de son pancréas font encore l'objet de nombreuses controverses. Pour ce type d'étude, deux modes d'approche ont été essentiellement utilisés. Le premier est l'induction d'un diabète permanent chez la rate gravide par injection d'alloxane ou de streptozotocine avant (Kervran et al., 1978 ; Prager et al., 1974 ; Pitkin et Van Horden, 1974) ou pendant (Aerts et Van Assche, 
1977 ; Golob et al., 1970) la gestation aboutissant généralement à une hyperglycémie sévère, en tout cas difficile à contrôler. De plus, les résultats obtenus par cette méthode sont contradictoires et semblent très dépendants de la dose de drogue injectée. L'autre mode utilisé consiste en l'injection unique ou la perfusion à court terme de glucose à la rate gravide, ceci plus particulièrement quand on s'attache à l'étude de l'insulino-sécrétion chez le fœetus (Kervran et Girard, 1974); il ne permet pas d'étudier les conséquences pour le fœtus d'une hyperglycémie prolongée.

Nous avons utilisé ici une technique (Nicolaïdis et al., 1974) permettant un contrôle aussi strict que possible de la glycémie maternelle en induisant chez la rate gravide non contrainte une hyperglycémie chronique et modulable par perfusion continue de glucose. Cette technique permet, en outre, de dissocier l'hyperglycémie des autres anomalies métaboliques dues au diabète.

Le but de ce travail a été d'évaluer chez le foetus de rat à terme les effets d'une hyperglycémie prolongée sur la croissance, les sécrétions du pancréas endocrine et sur le métabolisme glucidique dans le foie. Ces effets ont également été étudiés chez le fœtus post-mature, car des études précédentes avaient montré, d'une part que la prolongation de la gestation induisait une augmentation importante du rapport glucagon/insuline (Portha et al., 1978), et d'autre part que les modifications de la croissance et de l'adiposité fœtale liées à l'augmentation de la concentration plasmatique en insuline étaient d'autant plus nettes que I'on avait affaire à des stades de gestation plus avancés (Picon, 1967).

\section{Matériel et méthodes.}

Animaux. - Des rates albinos de souche Sherman élevées au laboratoire sont nourries avec un aliment solide (comprimé UAR B-03). Les mâles sont mis dans les cages des femelles 2 soirs par semaine vers $17 \mathrm{~h}$ et retirés le lendemain vers $9 \mathrm{~h}$; la gestation est déterminée par palpation, 14 jours plus tard. Dans notre élevage, la durée normale de la gestation est de 22 jours. La prolongation de la gestation est obtenue par l'administration quotidienne de $2,5 \mathrm{mg}$ de progestérone (Lutogyl, Roussel, France), injectée par voie sous-cutanée. Les rates sacrifiées à terme ne reçoivent aucun traitement.

L'administration de glucose aux rates gravides est effectuée par la technique de perfusion à long terme à des animaux non contraints de Nicolaïdis et al. (1974). Après anesthésie à la Kétamine (Imalgène, Mérieux, France $125 \mathrm{mg} / \mathrm{kg}$ IP), les rates reçoivent une sonde constituée d'un cathéter souple (Silastic, Dow Corning, USA) placée à demeure dans la jugulaire et reliée à une pièce de tube flexible (Tygon, Norton, USA) fixée au sommet du crâne à l'aide de ciment dentaire (Pennwalt, SS White, USA). Chaque rate est placée dans une cage cylindrique en plexiglas et reliée en permanence à une pompe péristaltique (Minipuls HP-2, Gilson, France). Un joint tournant étanche permet à l'animal de se mouvoir librement. Dans tous les cas, les rates gravides sont perfusées pendant 3 jours consécutifs avant le prélèvement des fœtus. La perfusion commence $24 \mathrm{~h}$, après l'implantation de la sonde. Du glucose stérile hypertonique à $30 \%$ (Chaix et 
Du Marais, France), est perfusé à raison de $39 \mathrm{ml} /$ jour. Les rates témoins reçoivent une perfusion d'eau distillée stérile (Chaix et Du Marais, France). Nous avons choisi l'eau distillée plutôt que du sérum physiologique, pour éviter une surcharge sodée trop importante. Vu le faible débit de la perfusion par rapport au flux de la circulation dans la veine cave supérieure, il n'était théoriquement pas à craindre d'accident hémolytique et nous n'en avons, de fait, jamais observé.

L'effet de bloquants spécifiques des récepteurs $\alpha$-(phentolamine) et $\beta$-(propanolol) des catécholamines sur la concentration plasmatique de l'insuline et du glucagon est apprécié sur certaines portées post-matures de 23,5 jours, après injection aux fœetus par voie sous-cutanée, à travers la paroi utérine, de $10 \mu \mathrm{g}$ de phentolamine ou de propanolol. Les fœtus témoins reçoivent une injection de $\mathrm{NaCl} 9 \%$.

Echantillons. - La glycémie des rates gravides perfusées est contrôlée quotidiennement sur des échantillons de sang prélevés à la queue. Après anesthésie (Imalgène $125 \mathrm{mg} / \mathrm{kg}$; IP) et laparotomie de la mère, les fœtus sont extraits successivement de l'utérus, en laissant le placenta et le cordon ombilical in situ de manière à ne pas interrompre les échanges fœto-maternels. Le sang est collecté après section des vaisseaux axillaires, le foie est rapidement prélevé et pesé, puis, soit congelé dans l'azote liquide pour le dosage ultérieur du glycogène, soit homogénéisé dans du tampon maléate $0,5 \mathrm{~mole} / \mathrm{l}(\mathrm{pH}=6,5)$ pour le dosage de la phosphoénolpyruvate carboxykinase (PEPCK). Les fœetus sont alors complètement extraits et pesés, le poids final tenant compte du poids du sang et du foie prélevés. Les fœtus sont conservés à $-20^{\circ} \mathrm{C}$ jusqu'à la détermination des lipides totaux. Pour évaluer le taux de lipogenèse dans la carcasse des fœetus, 6 rates gravides reçoivent, au stade de 23,5 jours, $10 \mathrm{mCi}$ de $3 \mathrm{H}_{2} \mathrm{O}$, par le système de perfusion, une heure avant l'ablation des fœtus. La lipogenèse est estimée par la méthode de Fain et al. (1965) et exprimée en umoles de ${ }^{3} \mathrm{H}_{2} \mathrm{O}$ incorporée dans les lipides $\mathrm{g}$. de tissu ${ }^{-1}$.heure ${ }^{-1}$. Le contenu en lipides de la carcasse est mesuré sur le fœtus entier selon la méthode de Folch et al. (1957).

Des aliquots de plasma de plusieurs fœetus de la même portée (3 à 4) sont déprotéinisés par l'acide trichloracétique à $5 \%$. Les surnageants trichloracétiques sont utilisés pour la détermination colorimétrique de l'azote $\alpha$-aminé (Malangeau et al., 1973), en utilisant la sérine comme standard. La glycémie est déterminée sur des échantillons de $10 \mu \mathrm{l}$ de plasma, à l'aide d'un microanalyseur de glucose (Beckman, Palo Alto, USA). L'insulinémie est mesurée en utilisant l'insuline de rat (Novo, Copenhague, Danemark) comme standard, de l'insuline porcine marquée par ${ }^{125}$ l comme traceur, et de l'anticorps anti-insuline humaine. L'hormone libre est séparée de l'hormone liée à l'aide de silicate de magnésium. La sensibilité de la méthode est de $6 \mu \mathrm{U} / \mathrm{ml}$ avec un coefficient de variation de $10 \%$ entre les différents dosages.

La glucagonémie est déterminée sur des échantillons de plasma sans extraction préalable en utilisant de l'anticorps anti-glucagon $30 \mathrm{~K}$, du glucagon marqué par ${ }^{125}$ I comme traceur, et du glucagon de porc comme standard (B66 K 1070, Dr J. Schlichtkrull, Novo, Copenhague, Danemark). Une préparation de charbon-dextran est utilisée pour séparer hormone libre et hormone liée. Le coefficient de variation entre les différents dosages est de $10 \%$. 
La concentration du glycogène hépatique est déterminée par la technique à l'amylo-glucosidase de Roehring et Allred (1974).

La PEPCK cytosolique est mesurée selon la technique de Ballard et Hanson (1967) sur le surnageant obtenu à partir des homogénats de foie. L'activité est mesurée par la conversion du $\mathrm{NaH}^{14} \mathrm{CO}_{3}$ (Radiochemical Centre, Amersham, Grande Bretagne) en malate exprimée en nmole. $\mathrm{h}^{-1} . \mathrm{mg}$ de protéine ${ }^{-1}$. La concentration en protéines est mesurée par la méthode de Lowry et al. (1951).

Les résultats sont exprimés sous forme de moyennes accompagnées de l'erreur standard de la moyenne. La signification statistique des différences observées entre les valeurs moyennes obtenues pour chaque lot d'animaux est évaluée par le test de Student pour les valeurs non appariées. Les corrélations statistiques entre 2 paramètres sont établies par la méthode des moindres carrés.

\section{Résultats.}

Les rates ayant reçu une perfusion de glucose présentent une hyperglycémie assez modérée (environ $2 \mathrm{~g} / \mathrm{l}$ ) tout au long de la période de perfusion. Leur glycémie est toujours très significativement supérieure à celle des rates témoins (fig. 1). Cette hyperglycémie s'accompagne chez les rates à terme comme chez les rates en gestation prolongée de modifications métaboliques et hormonales. L'insulinémie est plus élevée chez les rates hyperglycémiques et la glucagonémie fortement abaissée ; parallèlement, l'activité de la PEPCK hépatique est très diminuée et la concentration en glycogène hépatique très augmentée (tabl. 1). Par contre, la concentration plasmatique en azote $\alpha$-aminé n'est pas modifiée de manière significative par rapport aux rates témoins. La consommation alimentaire est très réduite chez les rates ayant reçu une perfusion de glucose $(15,6 \pm 0,2 \mathrm{Kcal} / \mathrm{j} ; \mathrm{n}=22)$ par rapport à celle des rates témoins $(67,3 \pm 1 \mathrm{Kcal} / \mathrm{j} ; \mathrm{n}=17)$.

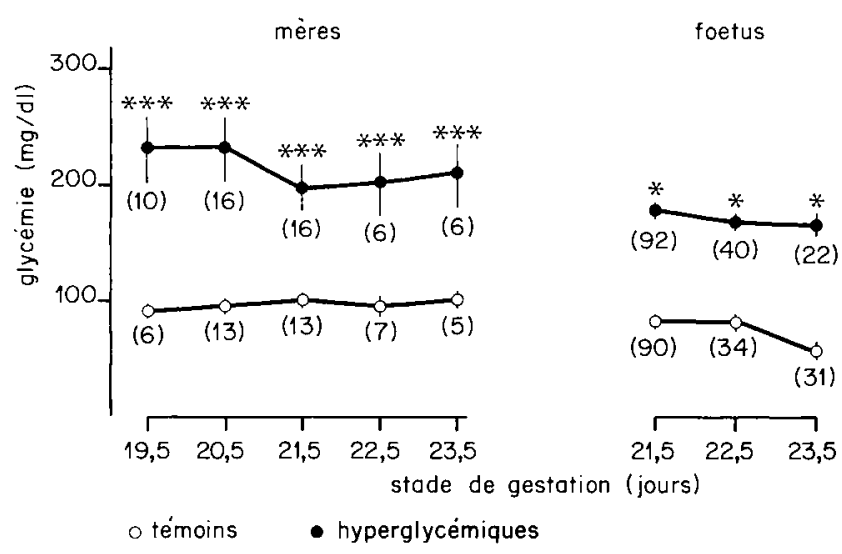

FIG. 1. - Evolution des glycémies maternelles et fotales. En ordonnée, la glycémie est exprimée en $\mathrm{mg} / \mathrm{dl}$. Le nombre de dosages est indiqué entre parenthèses.

*** $p<0,001$; différence statistique significative entre témoins et perfusés de glucose. 
Hyperglycémie et développement foetal

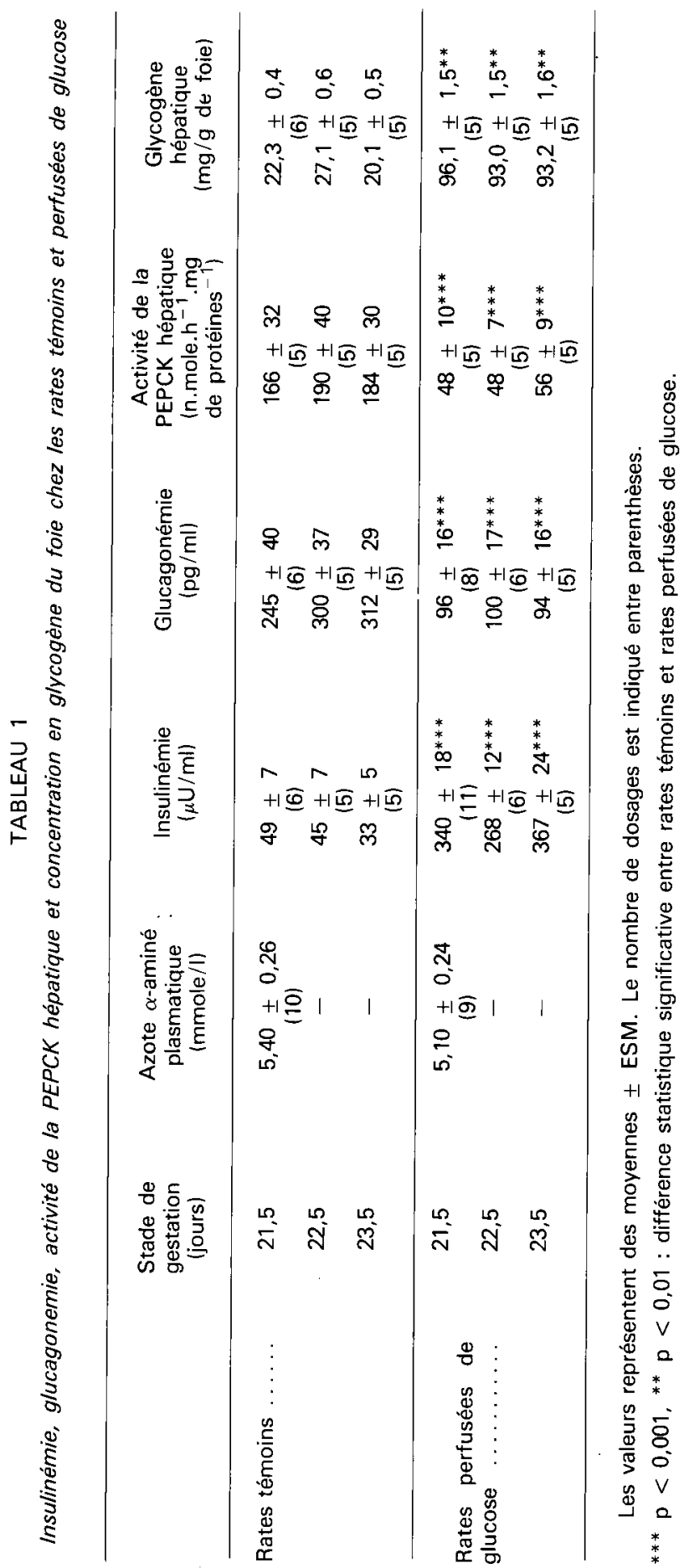

Reproduction, nutrition, développement $n^{\circ} 2 \mathrm{~B}$. 
La glycémie des fœtus de rates hyperglycémiques est très significativement supérieure à celle des fœus témoins indépendamment du stade de gestation (fig. 1). La concentration plasmatique en azote $\alpha$-aminé des fœtus hyperglycémiques $(11,9 \pm 0,6 \mathrm{mmole} / \mathrm{l} ; \mathrm{n}=12) \mathrm{n}$ 'est pas significativement différente de celle des foetus témoins $(12,1 \pm 0,6 \mathrm{mmole} / \mathrm{l} ; \mathrm{n}=12)$. Le niveau de l'insulinémie des fœtus à terme de mères ayant reçu une perfusion de glucose est nettement plus élevé que celui des fœetus témoins du même stade. Cette différence persiste chez les fœtus de 22,5 jours et de 23,5 jours, mais dans les 2 groupes, on assiste à une chute de l'insulinémie d'autant plus nette que la gestation est plus prolongée (fig. 2). La concentration plasmatique en glucagon est fortement abaissée chez les fœtus hyperglycémiques prélevés à terme par rapport aux fœtus témoins du même âge. Cependant, la concentration de cette hormone augmente de manière sensible avec la post-maturité chez les fœtus des 2 groupes (fig. 2). Ainsi le rapport glucagon/insuline augmente toujours au cours de la post-maturité mais de manière beaucoup plus importante chez les fotus de mères perfusées de glucose que chez les fœtus témoins (fig. 3). Au stade de 23,5 jours, l'insulinémie remonte fortement après l'injection de phentolamine dans les 2 groupes d'animaux alors que l'injection de propanolol reste sans effet (fig. 2). La glucagonémie n'est pas modifiée par l'administration de phentolamine, elle est diminuée par le propanolol, de façon non significative chez les fœtus témoins et de façon significative chez les fœtus hyperglycémiques. L'activité de la PEPCK hépatique est très faible chez les fœetus de 21,5 jours des 2 groupes. Pour les fœetus témoins, son activité augmente à 22,5 jours et surtout à 23,5 jours. Pour les fœtus de mères perfusées de glucose, l'activité de la PEPCK demeure inchangée à 22,5 jours, et double seulement à 23,5 jours (fig. 3). En outre, il existe une forte corrélation entre l'activité de la PEPCK et le rapport glucagon/insuline $(y=5,4 \times+36 ; n=100 ; r=0,9 ; p<0,001)$. L'accumulation de glycogène par le foie foetal n'est pas stimulée par la perfusion de glucose chez le fœetus à terme : $116 \pm 2 \mathrm{mg} / \mathrm{g}$ d'organe $(\mathrm{n}=15$ ) pour les fœtus hyperglycémiques, $110 \pm 2 \mathrm{mg} / \mathrm{g}$ d'organe $(\mathrm{n}=22$ ) pour les fœtus

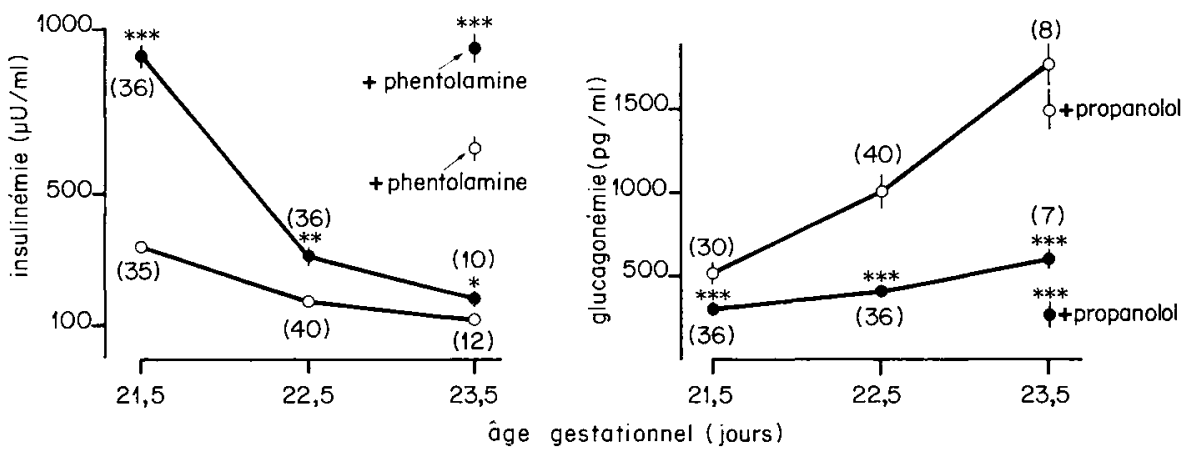

FIG. 2. - Insulinémie et glucagonémie chez les foetus à terme et postmatures. Le nombre de dosages est indiqué entre parenthèses.

${ }^{* * *} p<0,001 ;{ }^{* *} p<0,01 ;{ }^{*} p<0,05$; différence statistique significative entre témoins et perfusés de glucose.

$\mathrm{O}-\mathrm{O}=$ foetus témoins ; $-\bullet=$ fœtus de rates perfusées de glucose. 


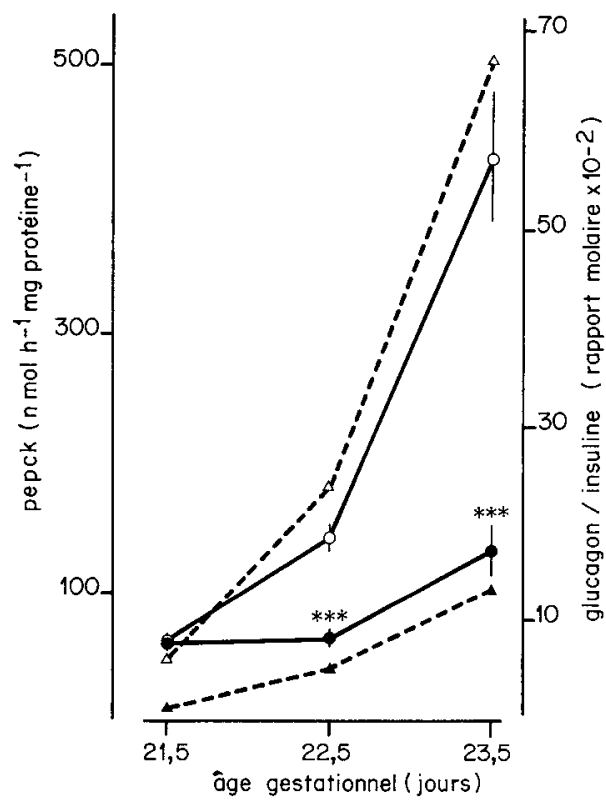

FIG. 3. - Evolution, au cours de la postmaturité, du rapport glucagon/insuline $(\Delta--\Delta=$ foetus témoins ; $\boldsymbol{\Delta}-\mathbf{\Delta}=$ foetus hyperglycémiques) et de l'activité de la PEPCK $10-0=$ fœtus témoins; - -0 fœutus hyperglycémiques). Chaque résultat représente la moyenne de 10 à 30 observations.

${ }^{* * *} p<0,001$; différence statistique significative entre témoins et perfusés de glucose.

témoins. Au stade de 22,5 jours, on n'enregistre aucune différence entre les 2 groupes : $91 \pm 4 \mathrm{mg} / \mathrm{g}$ d'organe $(\mathrm{n}=20$ ) pour les fotus hyperglycémiques, $85 \pm 2 \mathrm{mg} / \mathrm{g}$ d'organe ( $\mathrm{n}=25$ ) pour les foetus témoins. A 23,5 jours, la teneur en glycogène du foie est très diminuée chez les foetus témoins $(22 \pm 1 \mathrm{mg} / \mathrm{g}$ d'organe; $\mathrm{n}=35$ ) alors que l'épuisement de ces réserves est freiné chez les fœtus hyperglycémiques $(60 \pm 1 \mathrm{mg} / \mathrm{g}$ d'organe $; \mathrm{n}=22)$. Comme le montre la figure 4 , il existe une augmentation significative du poids corporel et du contenu en lipides de la carcasse chez les fœetus hyperglycémiques de 23,5 jours. Parallèlement, le taux de la lipogenèse dans la carcasse de ces fœtus est très augmenté (fig. 4).
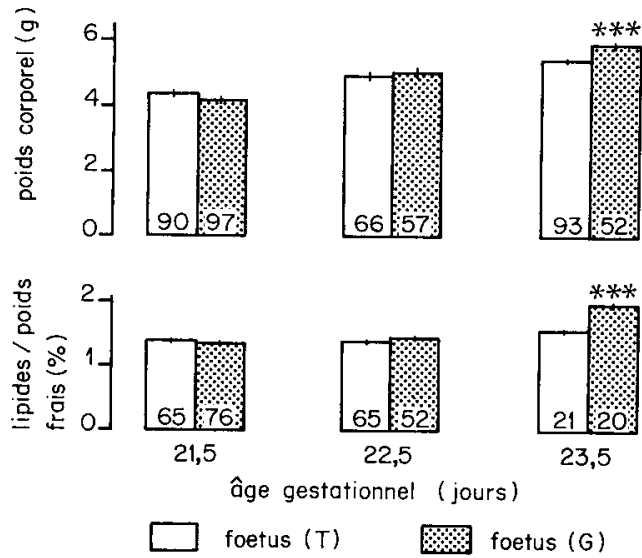

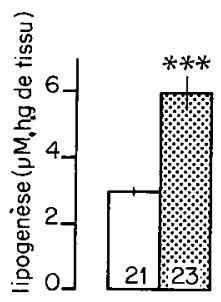

lipogenèse dans la carcosse des foetus de 23,5 jours

IG. 4. - Poids corporel, contenu en lipides de la carcasse chez les foetus à terme et postmatures. Taux de lipogenèse in vivo chez les fœtus de 23,5 jours. Nombre de dosages au bas des plots. ${ }^{* * *} p<0,001$; différence statistique significative entre témoins et perfusés de glucose. 


\section{Discussion.}

La technique utilisée nous a permis de provoquer une hyperglycémie modérée et contrôlable chez les mères et les fœtus. Les différents paramètres hormonaux et métaboliques étudiés chez les mères sont bien en rapport avec cette hyperglycémie. Comme ces paramètres ne varient que peu avec la prolongation de la gestation, on peut penser que l'injection de progestérone ne les a pas modifiés. La chute importante de la consommation alimentaire chez les rates perfusées de glucose, est en accord avec la théorie " glucostatique » de Jean Mayer (1955). Si on tient compte des calories apportées par la perfusion de glucose, il n'existe pas de différence notable entre rates ayant reçu une perfusion de glucose $(62 \mathrm{Kcal} /$ jour $)$ et rates témoins $(67 \mathrm{Kcal} /$ jour$)$. De plus, la concentration en azote $\alpha$-aminé plasmatique n'est modifiée ni chez les mères perfusées de glucose, ni chez leurs fœtus; ce qui suggère qu'une chute importante de l'apport alimentaire pendant les 3 derniers jours de la gestation n'empêche pas que l'apport global d'acides aminés aux fœtus soit inchangé, bien que la déficience d'un ou plusieurs acides aminés ne puisse être exclue.

L'augmentation de la glycémie des fœtus de mères ayant reçu du glucose reflète l'hyperglycémie maternelle; elle a eu des effets nets sur la concentration plasmatique des hormones pancréatiques. Les taux élevés d'insuline plasmatique chez les fœtus de mères hyperglycémiques confirment que la cellule $B$ fœtale est capable de répondre à une stimulation par le glucose ; ce qui est en accord avec les résultats de Kervran et Girard (1974), mais contredit d'autres études (Asplund et al., 1969 ; Clark et al., 1968). D'autre part, la glucagonémie chez les fœetus hyperglycémiques à terme est fortement abaissée. Des travaux précédents, fondés sur l'injection aiguë ou la perfusion à court terme de glucose, avaient conclu à l'insensibilité de la cellule $A$ du fotus de rat aux variations de la glycémie (Girard et al., 1973).

Notre étude montre que la cellule A fotale est sensible au glucose, à condition que la stimulation par le glucose soit prolongée. Comme l'avaient montré d'autres travaux (Portha et al., 1978), l'insulinémie décroît et la glucagonémie augmente au cours de la postmaturité dans les 2 groupes d'animaux. Ceci pourrait s'expliquer par une stimulation adrénergique et/ou une augmentation du taux des catécholamines circulantes au cours de gestations prolongées. La réponse du pancréas fotal à l'injection de phentolamine et de propanolol est bien compatible avec cette hypothèse. Les résultats obtenus montrent que l'inhibition partielle de la sécrétion d'insuline au cours de la postmaturité est due à l'activation de récepteurs $\alpha$-adrénergiques comme on la montré chez l'adulte (Porte, 1967), alors qu'ils suggèrent que la stimulation de la sécrétion de glucagon au cours de gestations prolongées passerait par l'activation de récepteurs $\beta$-adrénergiques, quoique, pour cette hormone, les données recueillies soient beaucoup moins nettes.

Ces modifications hormonales sont bien reflétées par les variations de l'activité de la PEPCK hépatique et nos résultats suggèrent que la chute du rapport glucagon/insuline peut inhiber partiellement l'induction de cette enzyme. Le fait que des fotus montrant des glycémies très proches présentent une activité de la PEPCK très différente et l'existence d'une corrélation étroite entre ce paramètre 
et le rapport glucagon/insuline, indiquent que I'induction de la PEPCK hépatique est provoquée plus par des variations de la concentration des hormones pancréatiques que par celle de la glycémie. D'ailleurs, des travaux précédents ont montré que des sucres comme le galactose et le mannose, capables de bloquer l'activité de la PEPCK sont aussi insulinosécréteurs (Lambert, 1970), alors que des sucres non-insulinosécréteurs comme le ribose, ou le 2-déoxyglucose sont sans effet sur l'activité de la PEPCK.

Chez les foetus à terme de mères perfusées de glucose, l'hyperglycémie et un rapport glucagon/insuline très bas n'ont pas eu pour effet d'augmenter significativement le stock de glycogène hépatique. II est connu qu'une accumulation importante de glycogène par le foie se produit dans les derniers jours de la gestation chez le fœetus de rat (Jost et Jacquot, 1958). II est possible qu'à terme, les capacités de stockage du foie soient à leur maximum et ne puissent être augmentées. La diminution du glycogène hépatique du stade 21,5 jours au stade 23,5 jours semble bien en rapport avec les modifications de la glycémie et du rapport glucagon/insuline ; la chute de la concentration en glycogène chez les fœtus témoins coïncide avec une augmentation importante du rapport glucagon/insuline alors que les fœtus hyperglycémiques du même âge, qui présentent une glycémie plus élevée et un rapport glucagon/insuline plus bas, voient leur glycogène hépatique diminuer de manière beaucoup moins importante.

En dépit de l'hyperinsulinisme très marqué présenté par les foetus hyperglycémiques à terme, ni le poids de ces fœtus à terme, ni la teneur en lipides de leur carcasse ne sont modifiés par rapport à ceux des fœtus témoins du même âge. Les résultats des travaux précédents relatifs au fœetus de rat à terme sont très contradictoires (cf. Introduction). Ceci contraste avec les observations concernant l'espèce humaine qui montrent une augmentation importante du poids et de la masse adipeuse sous-cutanée chez la plupart des nouveau-nés de mères diabétiques (Osler, 1960 ; O'Sullivan et al., 1966). Le facteur impliqué dans ce phénomène est selon Pedersen (Pedersen et al., 1954), la production excessive d'insuline par le foetus en réponse à l'hyperglycémie, ce qui entraînerait une augmentation importante de l'adiposité. On peut expliquer la discordance entre les résultats obtenus dans les 2 espèces par la différence dans la chronologie du développement du tissu adipeux chez le foetus de rat et le fotus humain. Chez celui-ci, le développement de ce tissu est déjà important in utero et il représente $16 \%$ du poids corporel à la naissance alors que chez le rat, le développement du tissu adipeux, qui ne représente à terme que $1 \%$ du poids corporel, est essentiellement post-natal. Nos résultats vont dans le sens d'une telle explication. En effet, l'augmentation de poids chez les foetus hyperglycémiques apparaît à 23,5 jours et la différence entre les 2 groupes expérimentaux devient très significative ; à cette augmentation de poids corporel correspond une élévation très nette des lipides totaux chez ces mêmes fotus. Ces résultats sont renforcés par la mesure de l'activité lipogénique in vivo, puisque nous observons une forte stimulation de la synthèse de lipides chez les fœtus de mères perfusées de glucose de 23,5 jours. 


\section{Conclusion.}

Les résultats obtenus au cours de cette étude indiquent que les cellules $B$ comme les cellules $A$ du pancréas fœtal de rat sont sensibles à une stimulation chronique par le glucose et qu'e les modifications hormonales entraînées par cette stimulation peuvent influer sur le métabolisme hépatique du glucose chez le fœtus. D'autre part, l'hyperinsulinisme chez les fœtus postmatures peut entraîner une augmentation significative du poids corporel et de l'accumulation des lipides; une situation qu'on peut rapprocher de celle des nouveau-nés de mères diabétiques.

$8^{e}$ Réunion du groupe Développement I.N.R.A., Tours, 12-13 mai 1982.

Remerciements. - Nous sommes très reconnaissants au Docteur Nicolaidis (Laboratoire de Physiologie Comportementale et Sensorielle, Collège de Francel de ses précieux et nombreux conseils concernant la technique de perfusion en continu, et au Docteur Rosselin (INSERM U.55) du don de I'insuline marquée à l'lode ${ }^{125}$. Ce travail a été financé en partie par un contrat de I'INSERM (Contrat de Recherche $n^{\circ} 817009$ ).

\section{Références}

AERTS L., VAN ASSCHE F. A., 1977. Rat foetal endocrine pancreas in experimental diabetes. J. Endocr., 73, 339-346.

ASPLUND K., WESTMAN S., HELLERSTRÖM C., 1969. Glucose stimulation of insulin secretion from the isolated pancreas of foetal and newborn rats. Diabetologia, 5, 260-262.

BALLARD F. J., HANSON R. W., 1967. Phosphoenolpyruvate carboxykinase and pyruvate carboxylase in developing rat liver. Biochem. J., 104, 866-871.

CLARK C. M. Jr., CAHILL G. F. Jr., SOELDNER J. S., 1968. Effects of exogenous insulin on the rate of fatty acid synthesis and glucose $\mathrm{C}^{14}$ utilization in the twenty day rat fetus. Diabetes, 17, 362-368.

FAIN J. N., SCOW R. O., URGOITI E. J., CHERNICK S. S., 1965. Effect of insulin on fatty acid synthesis in vivo and in vitro in pancreatectomized rats. Endocrinologv, 77, 137-149.

FOLCH J., LEES M., SLOANE-STANLEY G. H., 1957. A simple method for the isolation and purification of total lipids from animal tissues. J. biol. chem., 191, 497-509.

GIRARD J. R., CUENDET G. S., MARLISS E. B., KERVRAN A., RIEUTORT M., ASSAN R., 1973. Fuel hormones and liver metabolism at term and during the early postnatal period in the rat. J. clin. Invest., 52, 3190-3200.

GOLOB E. K., RISHI S., BECKER K. L., MOORE C., SHAH N., 1970. The effect of streptozotocin-induced diabetes on pancreatic insulin content of the fetuses. Diabetes, 19, 610-613.

JOST A., JACQUOT R., 1958. Sur le rôle de l'hypophyse des surrénales et du placenta dans la synthèse de glycogène dans le foie foetal de lapin et de rat. C.R. Acad. Sci. Paris, 247, 2459-2462.

KERVRAN A., GIRARD J. R., 1974. Glucose-induced increase of plasma insulin in the rat foetus in utero. J. Endocr., 62, 545-551.

KERVRAN A., GUILLAUME M., JOST A., 1978. The endocrine pancreas of the fetus from diabetic pregnant rat. Diabetologia, 15, 387-393.

LAMBERT A. E., 1970. Biochemical and morphological studies of cultured fetal rat pancreas. Th. Univ. Genève. 
LOWRY O. H., ROSENBROUGH N. Y., FARR A. L., RANDALL R. I., 1951. Protein measurement with the Folinphenol reagent. J. biol. Chem., 193, 265-275.

MALANGEAU P., BOURDON R., NICAISE A. M., MASSON B., 1973. Dosage des acides aminés dans les liquides de l'organisme. Ann. Biol. clin., 21, 3-13.

MAYER J., 1955. Regulation of energy intake and the body weight. The glucostatic theory and the lipostatic hypothesis. Ann. N. Y. Acad. Sci., 63, 15-42.

NICOLAÏDIS S., ROWLAND N., MEILE M. J., MARFAING-JALLAT P., PESEZ A., 1974. A flexible technique for long term infusions in unrestrained rats. Pharmacol. biochem. Behav., 2, 131-136.

OSLER M., 1960. Body fat of newborn infants in diabetic mothers. Acta endocr., 34, 227-286.

O'SULLIVAN J. B., GELLIS S. S., TENNEY B. O., 1966. Gestational blood glucose levels in normal and potentially diabetic women related to the birth weight of their infants. Diabetes, 15, 466-470.

PEDERSEN J., BOJEN-MOLLER B., POULSEN H., 1954. Blood sugar in newborn infants of diabetic mother. Acta endocr., 53, 310-314.

PICON L., 1967. Effect of insulin on growth and biochemical composition of the rat fetus. Endocrinology, 81, 1419-21.

PITKIN R. M., VAN HORDEN D. E., 1974. Fetal effects of maternal streptozotocin-diabetes. Endocrinology, 94, 1247-1253.

PORTE D., 1967. A receptor mechanism for the inhibition of insulin release by epinephrine in man. J. clin. invest., 46, 86-94.

PORTHA B., PICON L., ROSSELIN G., 1978. Postmaturity in the rat: high plasma glucagon levels in the fetus and the newborn. J. Endocr., 77, 153-154.

PRAGER R., ABRAMOVICI A., LIBAN E., LARON Z., 1974. Histopathological changes in the placenta of streptozotocin induced diabetic rat. Diabetologica, 10, 89-91.

ROEHRING K. L., ALLRED J. B., 1974. Direct enzymatic procedure for the determination of liver glycogen. Analyt. Biochem., 58, 414-421. 